\title{
Persistent Hypoglycemia in a Diabetic Patient
}

\section{Matthew W Jones DO, Rajib Bhattacharya MD}

University of Kansas Medical Center, Kansas City, KS

\section{Introduction}

Hypoglycemia occurs commonly in the diabetic patient. In some clinical situations, a hypoglycemic workup is indicated when concerned about an endogenous cause. Even so, iatrogenic causes of hypoglycemia in known diabetics must be ruled out to avoid unnecessary testing and therapy. In the setting of severe hypoglycemia, understanding the etiology is crucial as this could ultimately lead to death if untreated.

\section{Case Presentation}

HPI - A 69 year old African American male was admitted for persistent symptomatic hypoglycemia (dizziness, blurry vision, sweats, lethargy, and fatigue). $\mathrm{He}$ is a known diabetic of 6 years duration with good glycemic control. He was admitted to the hospital twice, within a 5 month time frame, with glucose levels in the 30's.

PMH/PSH - CAD, ICM, HTN, HLD, CKD, DM2,

Pacemaker placement

Social history - Single, Disabled, Former Smoker, No EtOH, No illicit drugs

Family History - Mother: DM2, Father: Heart Failure Medications - Plavix, Triamcinolone cream, Losartan, Carvedilol, Spironolactone, Furosemide, Aggrenox, Allopurinol, Omega-3 Fatty Acids, ASA, Ranitidine, Lovastatin, Nitroglycerin

Physical Exam - Hypotension, otherwise VSS, exam unremarkable other than $1+$ pitting edema in bilateral lower extremities

Labs - revealed low glucose, elevated creatinine, elevated BUN

Medication Reconciliation - An extensive medication reconciliation ensued, evaluating administration of insulin secretagogues (patient previously was on glimepiride). $\mathrm{He}$ and his family were adamant that he was not taking any glucose lowering agents as previously instructed at his last hospitalization for the same problem.

\section{Hospital Course}

-Persistent hypoglycemia despite multiple Dextrose 50 injections

-D10 drip started with and still with some persistent hypoglycemia. (Graph 1)

-A 72 hour fast was initiated, and the glucose trend while fasting is demonstrated in Graph 2. It was anticipated that the patient's glucose level would quickly drop when withholding food and D10.

- Initial glucose within normal range

- Quickly equilibrated and blood glucose unexpectedly began to rise.

- Pro-insulin, C-peptide, and insulin levels were drawn and all elevated at start of fast.

-After the fast was initiated the patient's family brought his medications from home for review.

- Glimepiride $4 \mathrm{mg}$ amongst his medication bottles.

- Admitted to usage for 2 days prior to admission.

- The fast was terminated and medication counseling for cessation ensued

\section{Graph: 1 On D10 Infusion}

Graph 2: Glucose during fast
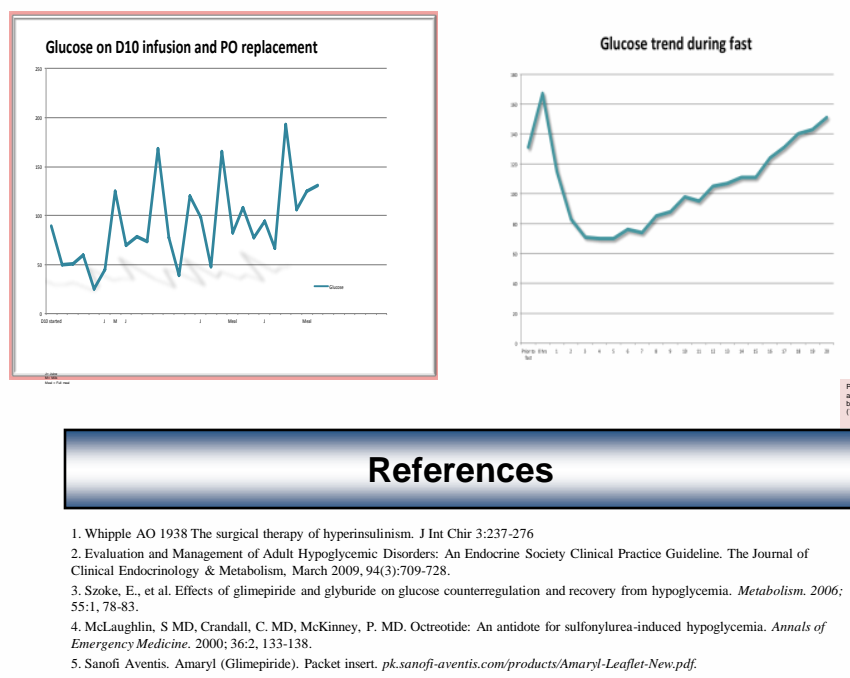

\section{Discussion}

Hypoglycemia in the diabetic patient:

Hypoglycemia in the diabetic patient is usually secondary to exogenous insulin and/or oral diabetic agents whether it be accidental, surreptitious, or malicious.

In this patient the use of exogenous agents was adamantly denied by patient and family, he had received counseling on this multiple times before. Therefore, the concern in an otherwise well individual would broaden the differential to include:

1) Insulinoma

2) Nesidioblastosi

3) Insulin autoimmune hypoglycemia

If Whipple's triad is present, a 72-hour fast is the diagnostic test of choice in determining the etiology of hypoglycemia. This involves monitoring of glucose levels and correlating levels of Insulin, Pro-insulin, C- Peptide, and Beta-hydroxybutyrate with hypoglycemic level.

\section{Sulfonylurea induced hypoglycemia}

Hypoglycemia is a well known side effect of oral sulfonylureas. In a study comparing glyburide to glimepiride, prevalence of more severe hypoglycemia with glyburide was suggested to be due to renal clearance. Even so, hypoglycemia remains a major side effect of Glimepiride.

Treatment:

The first intervention is cessation of the offending agent (in this case Glimepiride). Next is supportive treatment of hypoglycemia with dextrose infusions and carbohydrate rich oral intake with close glucose monitoring until medication has cleared. There has a been some efficacy Prewn with utilization of Octreotide as an antidote for sulfonylues.

Prevention:

Ultimately, prevention is the best intervention. Adequate counseling on proper use and, in this case, cessation should be offered, followed by close follow-up.

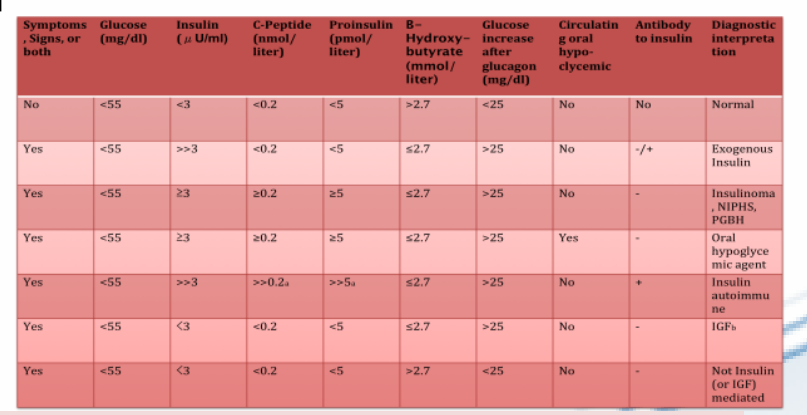

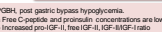

